\title{
Intranasal perillyl alcohol therapy improves survival of patients with recurrent glioblastoma harboring mutant variant for MTHFR rs 1801133 polymorphism
}

Giselle M. Faria ${ }^{1,2+} \mathbb{D}$, Igor D. P. Soares ${ }^{1 \dagger}$, Marcela D'Alincourt Salazar ${ }^{4}$, Marcia R. Amorim¹, Bruno L. Pessoa ${ }^{2,5}$, Clovis O. da Fonseca ${ }^{5}$ and Thereza Quirico-Santos ${ }^{1,2,3^{*}}$ (D)

\begin{abstract}
Background: Polymorphisms in MTHFR gene influence risk and overall survival of patients with brain tumor. Global genomic DNA (gDNA) methylation profile from tumor tissues is replicated in peripheral leukocytes. This study aimed to draw a correlation between rs 1801133 MTHFR variants, gDNA methylation and overall survival of patients with recurrent glioblastoma (rGBM) under perillyl alcohol (POH) treatment.

Methods: gDNA from whole blood was extracted using a commercially available kit (Axygen) and quantified by spectrophotometry. Global gDNA methylation was determined by ELISA and rs 1801133 polymorphism by PCR-RFLP. Statistical analysis of gDNA methylation profile and rs1801133 variants included Mann-Whitney, Kruskal-Wallis, Spearman point-biserial correlation tests (SPSS and Graphpad Prism packages; significant results for effect size higher than 0.4). Prognostic value of gDNA methylation and rs 1801133 variants considered survival profiles at 25 weeks of $\mathrm{POH}$ treatment, having the date of protocol adhesion as starting count and death as the final event.

(Continued on next page)
\end{abstract}

\footnotetext{
* Correspondence: tquirico@id.uff.br

${ }^{\dagger}$ Giselle M. Faria and Igor D. P. Soares contributed equally to this work. 'Instituto de Biologia, Universidade Federal Fluminense, Niteroi, Rio de Janeiro, ZC 24020-141, Brazil

${ }^{2}$ Programa de Pós-graduação em Neurologia, Faculdade de Medicina, Universidade Federal Fluminense, Niteroi, Rio de Janeiro 24020-141, Brazil Full list of author information is available at the end of the article
}

(c) The Author(s). 2020 Open Access This article is licensed under a Creative Commons Attribution 4.0 International License, which permits use, sharing, adaptation, distribution and reproduction in any medium or format, as long as you give appropriate credit to the original author(s) and the source, provide a link to the Creative Commons licence, and indicate if changes were made. The images or other third party material in this article are included in the article's Creative Commons licence, unless indicated otherwise in a credit line to the material. If material is not included in the article's Creative Commons licence and your intended use is not permitted by statutory regulation or exceeds the permitted use, you will need to obtain permission directly from the copyright holder. To view a copy of this licence, visit http://creativecommons.org/licenses/by/4.0/. The Creative Commons Public Domain Dedication waiver (http://creativecommons.org/publicdomain/zero/1.0/) applies to the data made available in this article, unless otherwise stated in a credit line to the data. 
(Continued from previous page)

Results: Most rGBM patients showed global gDNA hypomethylation (median = 31.7\%) and a significant, moderate and negative correlation between TT genotype and gDNA hypomethylation (median $=13.35 \%$; rho $=-0.520 ; p=0.003$ ) compared to CC variant (median $=32.10 \%$ ), which was not observed for CT variant (median $=33.34 \%$; rho = -0.289 ; $p=0.06$ ). gDNA hypermethylated phenotype (median $=131.90 \%$ ) exhibited significant, moderate and negative correlations between $\Pi$ genotype (median $=112.02 \%$ ) and gDNA hypermethylation levels when compared to CC ( median $=132.45 \%$; rho $=-0,450 ; p=0.04$ ) or CT (median $=137.80 \%$; rho $=-0.518 ; p=0.023$ ) variants. $\Pi$ variant of rs1801133 significantly decreased gDNA methylation levels for both patient groups, when compared to CC (d values: hypomethylated = 1.189; hypermethylated $=0.979$ ) or $C T$ ( $d$ values: hypomethylated $=0.597$; hypermethylated $=1.167$ ) variants. Positive prognostic for rGBM patients may be assigned to gDNA hypermethylation for survivors above 25 weeks of treatment (median = 88 weeks); and TT variant of rs 1801133 regardless $\mathrm{POH}$ treatment length.

Conclusion: rGBM patients under $\mathrm{POH}$-based therapy harboring hypermethylated phenotype and $\Pi$ variant for rs 1801133 had longer survival. Intranasal POH therapy mitigates detrimental effects of gDNA hypomethylation and improved survival of patients with rGBM harboring TT mutant variant for MTHFR rs 1801133 polymorphism.

Trial registration: CONEP -9681- 25,000.009267 / 2004. Registered 12th July, 2004.

Keywords: Perillyl alcohol, Glioblastoma, Methylation, Polymorphism, MTHFR, Epigenetics

\section{Background}

DNA methylation status, folate metabolism and its relevance for glioblastoma patients under perillyl alcohol (POH) inhaled therapy

Similarities on global DNA methylation status among different tissues within the same individual indicate genetic influence on genomic DNA methylation status [1]. Genome-wide changes in DNA methylation pattern is a hallmark of cancer, particularly glioblastoma (GBM) [2]. GBM is the most common and highly proliferative brain tumor in adults. Genome-wide DNA hypomethylation in GBM supports an aggressive tumor phenotype by promoting genomic instability and oncogene activation, while loci-specific DNA hypermethylation silences tumor suppressor genes [3]. Epigenetic alteration in genomic DNA (gDNA) is a reversible and early event in malignant transformation, regulating gene expression and genomic stability [4]. Importantly, the reversible nature of gDNA methylation upon folate supplementation is being evaluated as a possible therapeutic strategy in animal models of glioma [3]. Nonetheless, prognosis of GBM patients remains dismal. Clinical presentation is often associated to functional damage caused by anatomical location of the tumor in the brain tissue; partly because tumor lesions may remain undetectable by magnetic resonance image for many years [5-8]. Despite recent advances on neuroimaging and therapeutic approaches, time to tumor recurrence in GBM patients treated with surgery followed by chemo-radiotherapy is only 25 to 40 weeks, with a median overall survival around 14 months after diagnosis [9-12].Therefore, following the emergence of drug-resistant clones, virtually all patients with GBM will suffer disease recurrence [13-15].
The naturally occurring monoterpene perillyl alcohol $(\mathrm{POH})$ halts tumor progression and prolongs the overall survival in patients with treatment-refractory brain tumors [16-18]. Such encouraging findings result from the intranasal administration of $\mathrm{POH}$, thus assuring the delivery of a pleiotropic compound with cytotoxic, pro-apoptotic, anti-inflammatory and antiangiogenic properties directly into the brain tissue. Moreover, delivery of $\mathrm{POH}$ through the intranasal route may avoid the hepatic first passage effect and maximize the direct antitumor effect of the treatment against malignant brain tumors [18-20].

Polymorphisms on the gene encoding the methylenetetrahydrofolate reductase (MTHFR) enzyme, which plays a key role in the folate-mediated one-carbon metabolism, also affects proliferation rates and disease progression, as tumor cells frequently rely on the folate metabolism as a major source of one-carbon units for anabolic processes, nucleic acid synthesis and DNA methylation [13, 21-26]. Functional polymorphism rs1801133 (C677T) in the MTHFR gene consists of a cytosine $(\mathrm{C})$ to thymidine $(\mathrm{T})$ transition at the nucleotide 677 , and results in an aminoacid change from alanine to valine at the encoded protein. Such change results in an enzyme with decreased activity due to its thermolabile property [27] and metabolic relevance both in the physiological highly anabolic brain parenchyma and in the highly metabolic demanding GBM context [28].

Recently, we demonstrated that levels of circulating molecular markers (cell-free DNA) reflect brain tumor activity [29] being a noninvasive screening tool for early tumor recurrence [30], prognostic evaluation and response to $\mathrm{POH}$ treatment [29].Since abnormalities on genomic DNA (gDNA) methylation is considered to be 
an early and reversible event of malignant transformation, the present study aimed to investigate the association between global gDNA methylation levels with functional polymorphism rs1801133 (C677T) of the MTHFR gene and survival of patients with recurrent GBM (rGBM) undergoing POH-base therapy.

\section{Methods}

\section{Study population}

Before adhering to the protocol, all patients and/or his /her legal representative along with their primary care physician received detailed information about the study and agreed to participate in the Phase I/II study to evaluate the therapeutic efficacy of intranasal administration of the monoterpene perillyl alcohol $(\mathrm{POH})$ by signing an informed consent form. As a criterion for inclusion in the $\mathrm{POH}$ protocol, a diagnosis of recurrent malignant GBM was required, based on the initial diagnosis from the histopathological and image findings, as well as the presence of detectable and contrastingenhancing mass provided by magnetic resonance imaging (MRI) at the time of protocol inclusion. All patients included in this study had already failed all forms of conventional therapy (surgery, radiotherapy and specific chemotherapy for brain tumors) and were receiving symptomatic and palliative care. Retrospective data from one hundred adult patients with a proven diagnosis of recurrent GBM (rGBM) were considered by this study: 38 females, median age 50 years-old (range: 18 to 78 years) and 62 males, median age 52 years-old (range: 19 to 76 years).

$\mathrm{POH}$ was formulated to be used by inhalation by the University Pharmacy in accordance with international patent application US Patent Application 20,040,087,651 May 6, 2004 and Brazilian PI0107262-5 with approval by local Board of Health. The work followed the norms of the Helsinki Convention for clinical study and was approved by local research ethics committee (CONEP registration $9681 \quad \mathrm{n}^{\circ}$. 25000.009267 / 2004; CAAE0085.0.258.000-08).

\section{Extraction and quantification of genomic DNA}

Extraction of genomic DNA obtained from the peripheral blood of rGBM patients followed the protocol described in the AxyPrep Blood Genomic DNA Miniprep kit (Axygen). Genomic DNA resulting from the extraction of whole blood was quantified by spectrophotometry (NanoVue Plus; GE Healthcare Life Sciences), and stored at $-20^{\circ} \mathrm{C}$ until the moment of use.

\section{Global gDNA methylation levels}

Global methylation levels were determined from $30 \mu \mathrm{L}$ of DNA (200 ng) through the Imprint (Methylated DNA Quantification Kit, Sigma Chem Co., USA), following the manufacturer protocol. Since 70 to $90 \%$ of human genomic DNA obtained from somatic cells is considered to be methylated, and that DNA hypomethylation consists of a hallmark of malignant transformation [31-33] we established $90 \%$ as the experimental cut-off value for differentiation of both gDNA methylation phenotypes.

\section{Genotyping analysis}

Genotyping of the $677 \mathrm{C}>\mathrm{T}$ polymorphism in the MTHFR gene was performed by the PCR-RFLP (Restriction Fragment Length Polymorphism) method using the following primers: forward:

5' GAAGCAGGGAGCTTTGAGGCTGACCT-3' and reverse: 5'-AGGGATGCCCATGTCGGTGCATGCCT-3 'Amplifications were performed using a Veriti ${ }^{\circ}$ 96-well thermal cycler (Applied Biosystems, USA) under the following conditions: initial denaturation at $94{ }^{\circ} \mathrm{C}$ for $4 \mathrm{~min}$, 35 cycles of $30 \mathrm{~s}$ at $94^{\circ} \mathrm{C}$, annealing at $65^{\circ} \mathrm{C}$ for $30 \mathrm{~s}$ and extension at $72{ }^{\circ} \mathrm{C}$ for $30 \mathrm{~s}$, followed by a final extension step of $72^{\circ} \mathrm{C}$ for $5 \mathrm{~min}$. For complete digestion of fragments, $5 \mu \mathrm{L}$ of the PCR product was incubated at $65^{\circ} \mathrm{C}$ for $3 \mathrm{~h}$. A single fragment of 142 base pairs (bp) was identified as homozygous (CC) for the MTHFR normal allele; individuals bearing the $677 \mathrm{~T}$ polymorphic allele have a restriction site for the TaqI enzyme, which digests the $142 \mathrm{bp}$ fragment at 58 and $84 \mathrm{bp}$ and a threefragment pattern of 142,84 , and 58 bp were identified as heterozygous genotype $(\mathrm{CT})$ [34]. The restriction sites profile was visualized on $3 \%$ agarose gel using GelRed ${ }^{m}$ as a dye. For each digestion and enzymatic digestion verification carried out by electrophoresis, at least one previously confirmed polymorphic positive homozygote control was used.

\section{Statistical analysis}

Mann-Whitney test was selected for comparison of two independent groups according to methylation phenotypes (hypomethylated or hypermethylated). Pearson Chi-square test was chosen to evaluate the HardyWeinberg (HWE) equilibrium and also to perform comparison of proportions of genotypes, followed by Bonferroni correction (significant results for adjusted $p<0.02$ ). Kruskal-Wallis followed by pairwise comparison tests were employed to compare methylation median according to different genotypes of $r s 1801133$ (C677T), significant results for adjusted $p<0.02$ ). Effect size estimation (d value) was performed in addition to multiple comparison tests to highlight the magnitude of the relationship of variables evaluated, when significant results were considered for $d \geq 0.4$, related to the zone of desired effects) $[35,36]$. Spearman point-biserial correlation was selected to evaluate the relationship between continuous and categorical variables (genotypes and global gDNA methylation levels) [37].Prognostic value of both gDNA 
methylation phenotypes and $r s$ 1801133MTHFR variants was performed considering 25 weeks (cut-off) as critical reference time for $\mathrm{POH}$ treatment evaluation, considering the historical survival data of rGBM patients that fail response to standard therapeutic scheme [12]. For such analysis, the date of protocol adhesion was considered as the starting point and the date of death as final event $(n=67)$. Patients who abandoned the study protocol were not considered for such evaluation $(n=33)$. All the above-mentioned statistical tests were run at SPSS 20.0 and GraphPad Prism 5.0 softwares.

\section{Results}

\section{rGBM patients with hypomethylated gDNA and worse} prognosis during $\mathrm{POH}$ inhaled therapy

Global DNA methylation status of rGBM patients were determined using gDNA extracted from peripheral blood mononuclear cells. Patients were assigned to the hypomethylated group if individual global gDNA methylation levels were $\leq 90 \%$; and to the hypermethylated group if gDNA methylation levels were $>90 \%$ [31]. The majority (67\%) of the rGBM patients presented with hypomethylated gDNA (median $=31.7 \%$; range: 3.4 to $87.0 \%$ ) vs. the hypermethylated group (33\%; median $=131.9 \%$; range: 90.4 to $206 \%)$, with a significant difference $(p<0.0001$; $\mathrm{d}=2.766$ ) between both groups (Fig. 1a).

Since gDNA methylation abnormalities are a hallmark of tumor progression [2], we proceeded to evaluate the impact of gDNA methylation status on the overall survival of patients undergoing $\mathrm{POH}$-based treatment considering 25 weeks as survival time cutoff value. Indeed, the multiple comparison tests performed at this specific time point for each distinct gDNA methylation phenotypes (Fig. 1b; Supplementary Table 1) showed significant differences between such groups $(p<0.0001 ; \mathrm{d}=$ 3.234). Pair wise comparisons showed significant differences $(d=0.639)$ in terms of median survival times between gDNA hypomethylated (44 weeks) and hypermethylated phenotypes (88 weeks) for those rGBM patients with survival times higher than 25 weeks. Conversely, rGBM patients who survived less than 25 weeks did not present median survival differences $(p=0.811$; $\mathrm{d}=0.093$ ) despite of significant distinct gDNA methylation profiles $(41.40 \%$ vs $135.98 \% ; \quad p<0.0001, \quad d=$ 2.941).Significant differences were observed for median survival profiles within hypomethylated $(<25$ weeks $=11$ weeks; $>25$ weeks $=44$ weeks; $p<0.0001, \mathrm{~d}=2.991)$ and within hypermethylated ( $<25$ weeks $=12$ weeks; $>25$ weeks $=88$ weeks; $p<0.0001, d=3.00)$ groups. Finally, median survival profiles above or below 25 weeks were significantly different according both gDNA methylation phenotypes. Such results indicate longer survival times for rGBM survivors above 25 weeks, despite significant differences on gDNA methylation profiles.

\section{The rs 1801133 (C677T) MTHFR TT variant is associated with lower gDNA methylation levels}

Given the role of MTHFR C677T polymorphism to sustain anabolic pathways in rapidly proliferating tumor cells, we proceeded to evaluate the frequency of rGBM patients harboring different rs1801133 (C677T) genotypes, and to assess whether individual MTHFR C677T variants correlate with both gDNA methylation patterns. Genotype analysis of rGBM patients showed a

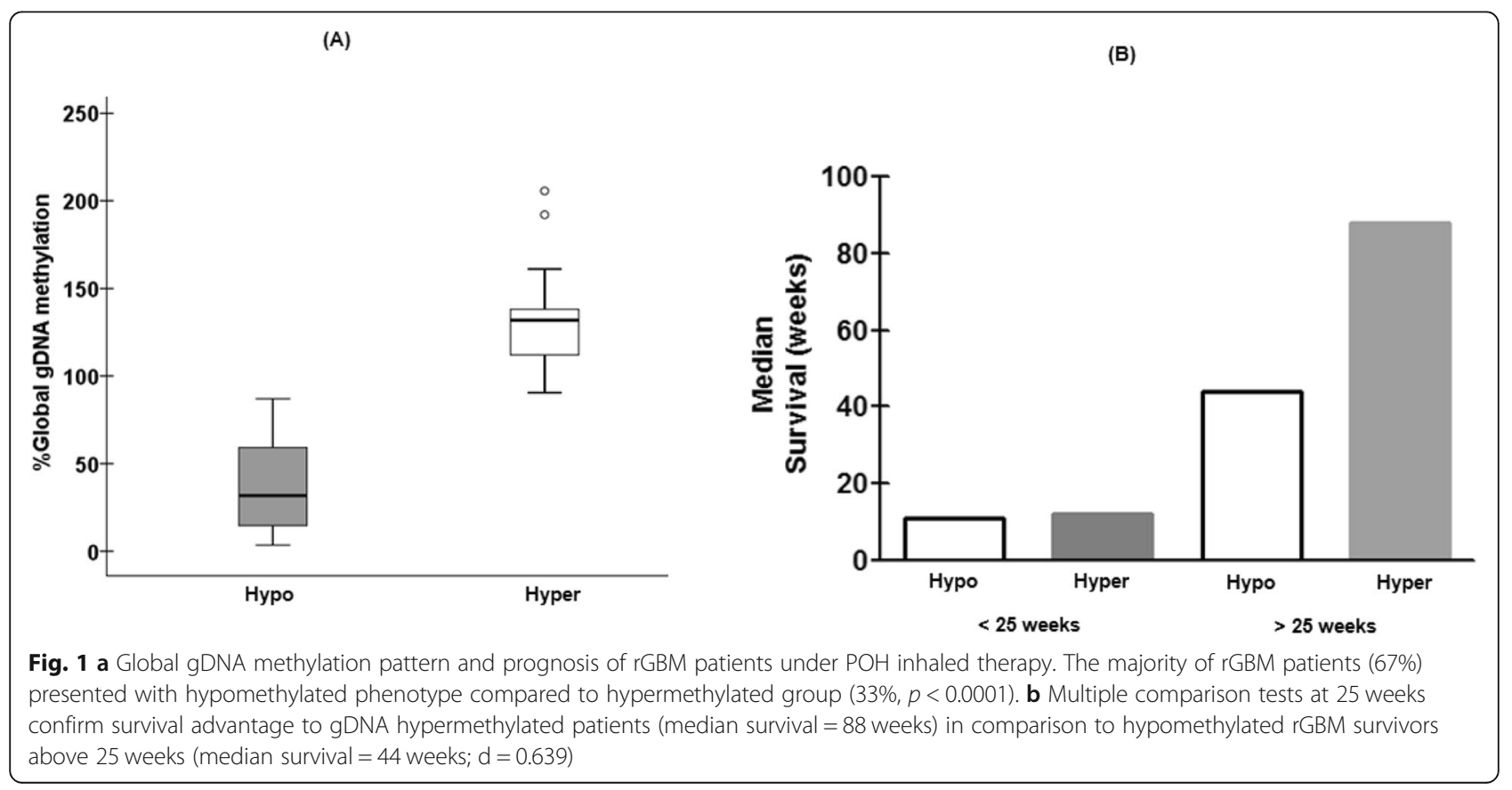


compliance with HWE $(p=0.65)$. We observed homozygous CC genotype in $38 \%$ of rGBM patients, while $49 \%$ of the patients carry the heterozygous CT genotype, and the remaining $13 \%$ carry the TT genotype. The comparison of different proportions of each genotype showed significant differences between observed frequencies for genotypes CC and TT $(p=0.003)$, and CT and TT $(p<0.0001)$.

Multiple comparison test showed significant differences between gDNA hypomethylation median results according each MTHFR genetic variant ( $\mathrm{d}$ value of Kruskal-Wallis test $=0.570$, Fig. 2a, Supplementary Table 2). Compared to rs 1801133 CC genotype, rGBM patients with the TT genotype presented with a 2.4-fold lower level of gDNA hypomethylation [median $=13.35 \%$ (range: 5.32 to $45.4 \%$ ) vs. median $=33.34 \%$ (range 11.7 to $87 \%$ ), respectively; $p=0.005, \mathrm{~d}=1.189$, Fig. 2a, Supplementary Table 2)]. The same magnitude (2.4-fold) upon gDNA hypomethylation reduction was observed between TT and CT rs 1801133 variants [median $=13.35 \%$ (range: 5.32 to $45.4 \%$ ) vs. median $=32.10 \%$ (range 3.4 to $84.8 \%$ )], respectively; $d=0.597$, Fig. 2a, Supplementary Table 2)]. In addition, we observed a significant, moderate and negative correlation between the TT genotype and gDNA hypomethylation compared to $\mathrm{CC}$ (rho $=-0.520$; $p=0.003$, Fig. 2b) but a non-significant, weak and

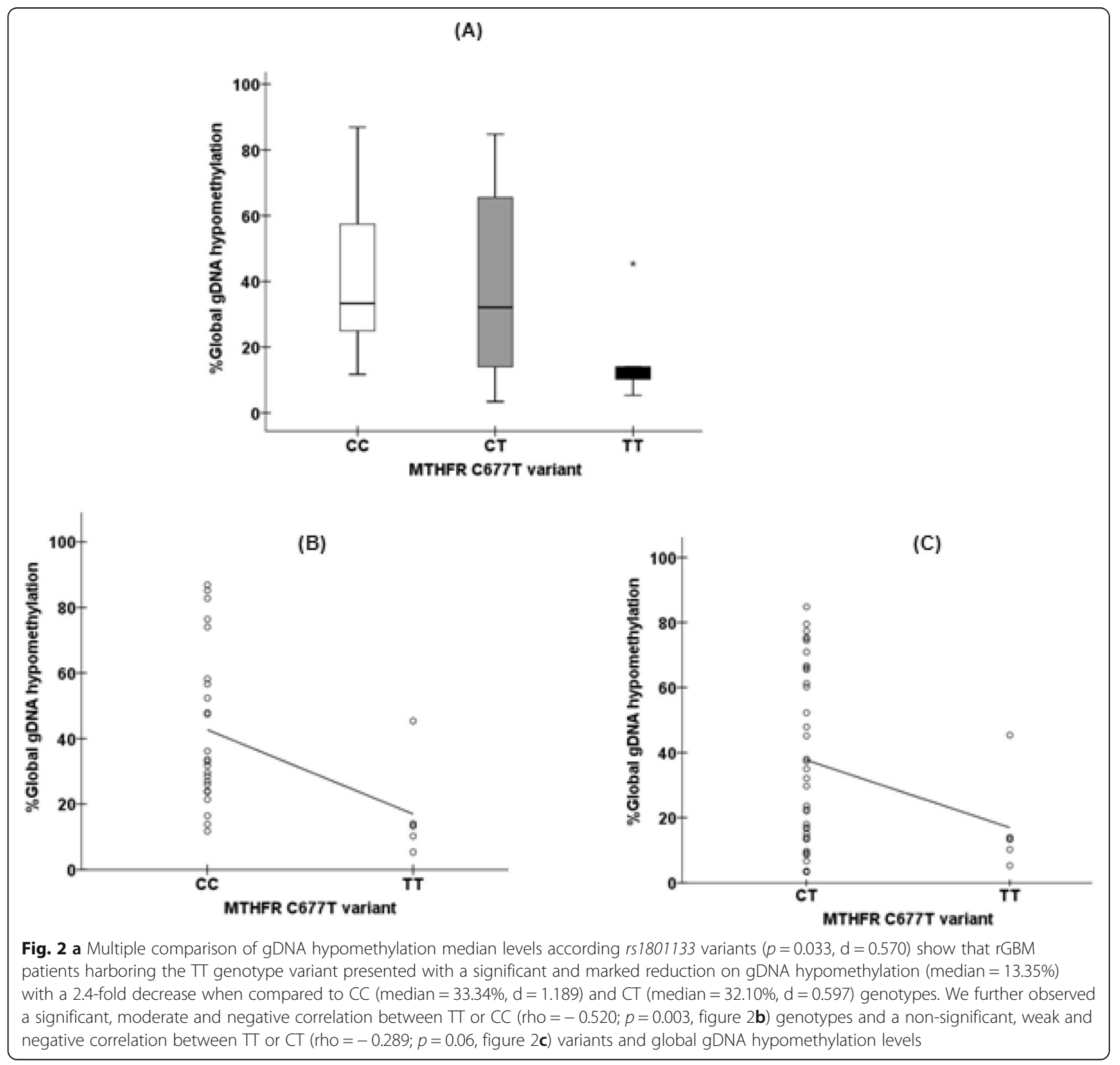


negative correlation between TT variant and gDNA hypomethylation (rho $=-0.289 ; p=0.06$, Fig. 2c) when compared tothe $\mathrm{CT}$ variant.

Regarding hypermethylated phenotype, multiple comparison test between rs1801133 variants showed significant differences on median gDNA methylation levels ( $d$ value for Kruskal-Wallis test $=0.751$, Fig. 3a, Supplementary Table 3). gDNA hypermethylation profile from individuals carrying the rs1801133 TT variant showed a significant 1.2-fold decrease in hypermethylation levels [median $=112.02 \%$ [range: 97.5 to $130.20 \%$ ] compared to CC genotype [median $=132.45 \%$ [range 90.4 to $192.03 \%$ ]; $d=0.979$, Fig. 3a] and to CT genotype [median $=137.80 \%$ [range 92.7 to 206\%]; $\mathrm{d}=1.167$, Fig. 3a]. In addition, we further observed significant, moderate and negative correlations between the TT genotype and gDNA hypermethylation compared to CC (rho $=-0.450 ; p=0.040 ;$ Fig. $3 \mathrm{~b})$ and CT $(\mathrm{rho}=-0.518$; $p=0.023$; Fig. 3c) genotypes.

\section{Survival analysis of rGBM patients under POH therapy according to different rs 1801133 (C677T) genotypes} Taking into account survival differences between both gDNA methylation groups at a specific time during treatment course ( 25 weeks, Fig. 1), and the impact of rs1801133 (C677T) polymorphic variants on gDNA

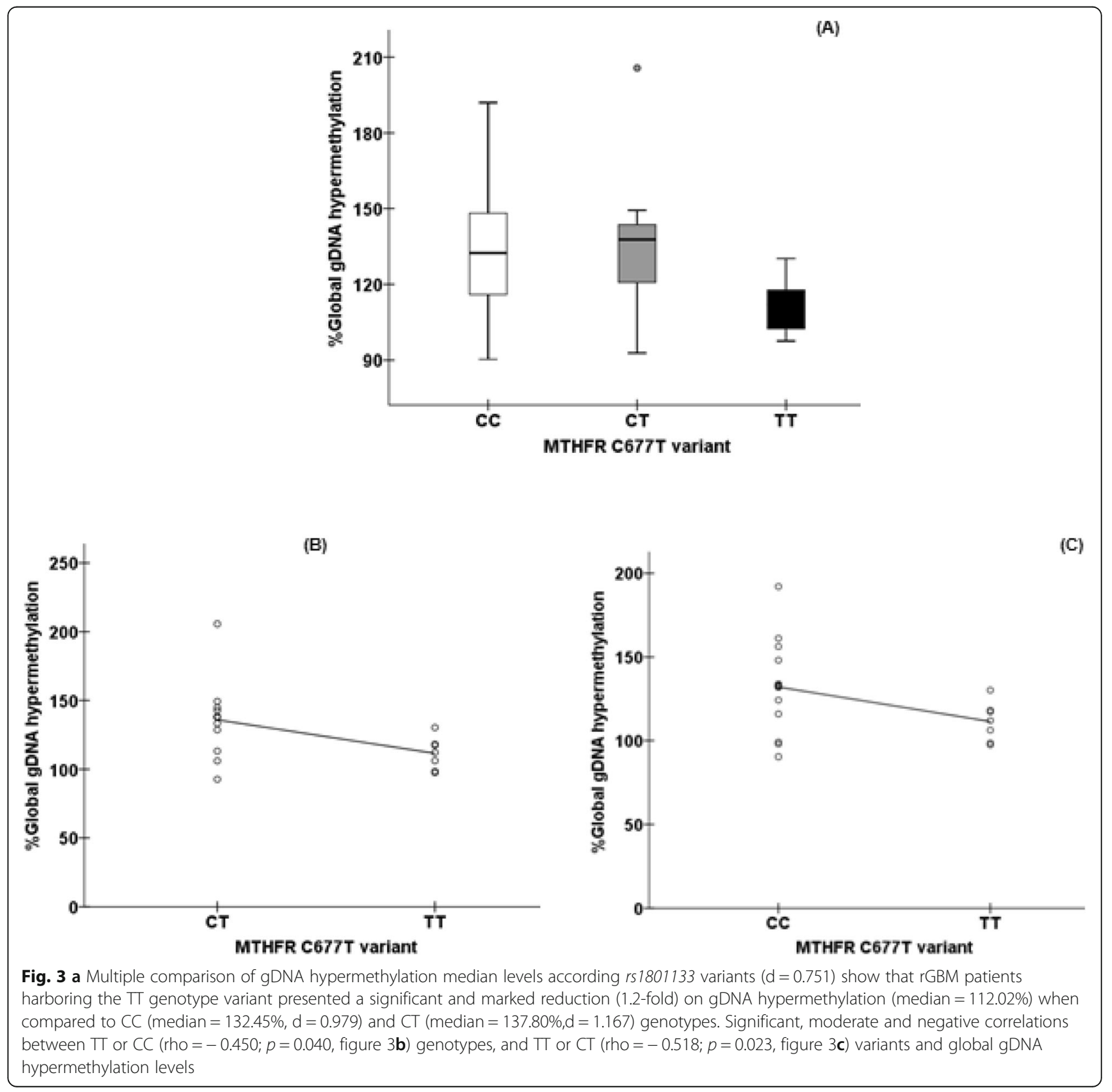


methylation levels (Figs. 2 and 3), we considered relevant to evaluate the relationship between these polymorphic variants and survival at 25 weeks for our rGBM cohort. Indeed, multiple comparison tests showed significant differences between MTHFR rs1801133 variants for those rGBM survivors who lived more or less than 25 weeks $(p<0.0001 ; \mathrm{d}=3.247)$. Pair wise comparison showed that rGBM patients with TT variant had better prognosis (18 weeks) when compared to $\mathrm{CC}$ or CT variants for those patients with lower survival $(\mathrm{CC}=8$ weeks; $\mathrm{d}=1.583$ and $\mathrm{CT}=12$ weeks, $\mathrm{d}=0.941$ ) and for those with higher survival $(\mathrm{TT}=92$ weeks; $\mathrm{CC}=44$ weeks; $\mathrm{d}=$ 0.648 and $\mathrm{CT}=48$ weeks, $\mathrm{d}=0.807$ ). It was also observed significant survival differences within and between MTHFR rs 1801133 variants after and before the selected median time ( 25 weeks) of survival after tumor recurrence (Supplementary Table 4, Fig. 4).

\section{Discussion}

Currently available treatments for rGBM are unable to effectively overcome the high intratumoral cellular and molecular heterogeneity to elicit sustained tumor regression. Disease complexity may be further enhanced by detrimental effects of cytotoxic chemotherapy and ionizing radiation on genetic and epigenetic stability $[15,22$, 26].Since tumor-associated changes in the gDNA methylation status is replicated in gDNA obtained from peripheral blood cells of cancer patients [38-40], analysis of gDNA global methylation status using peripheral mononuclear cells from rGBM patients could be potentially used as a non-invasive prognostic biomarker, representing a valuable circulating screening tool that better represents the dynamic nature of such intracranial malignancy [41].

Besides, the brain parenchyma under physiological conditions, presents a highly anabolic metabolism, where one-carbon units consist of key elements for macromolecules synthesis, gene expression control and redox balance [28]. The recognized impact of $r s 1801133$ (C677T) functional polymorphism impairment on the key regulatory enzyme (MTHFR) of folate metabolism gains increased relevance in the context of highly invasive and proliferative glioblastoma, especially if considered the tumor cells metabolic reprogramming as a key pillar of malignant transformation [42] [43, 44].

In the present study, we used gDNA isolated from peripheral blood samples to examine whether global gDNA methylation status and more specifically, rs1801133 polymorphism of the MTHFR gene, correlated with survival outcomes of rGBM patients undergoing intranasal $\mathrm{POH}$ treatment. All rGBM patients enrolled in this study were at terminal stage, after failing response to all therapeutic schemes (standard and second line therapies) and just receiving support and palliative care. In agreement with previous reports, we observed that the majority of rGBM patients presented wide gDNA hypomethylation [45]. Despite the significant survival differences for both methylation groups (44 weeks for hypomethylated vs 88 weeks for hypermethylated gDNA) after a specific moment of treatment ( 25 weeks), the present study

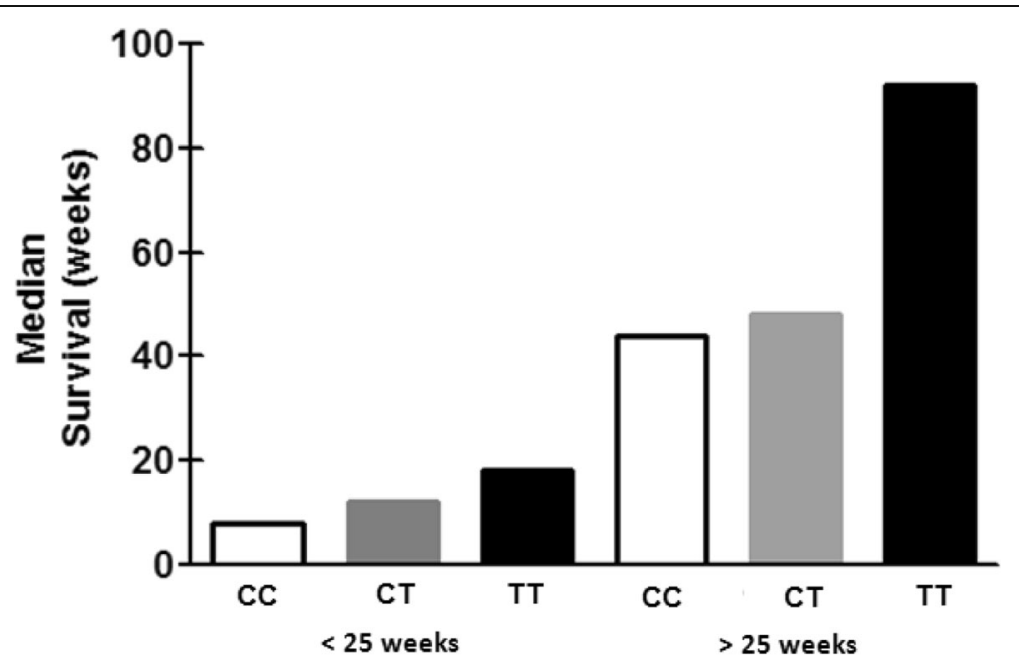

MTHFR C677T variant

Fig. 4 Multiple comparison tests at 25 weeks confirm positive prognosis to rGBM patients harboring the $\Pi$ polymorphic variant of MTHFR rs 1801133 in comparison to the remaining variants, both for patients with lower survival times $(T T=18$ weeks; $C C=8$ weeks; $d=1.583$ and $C T=$ 12 weeks, $d=0.941)$, as well as for those who achieved higher survival times ( $T=92$ weeks; $C C=44$ weeks; $d=0.648$ and $C T=48$ weeks, $d=$ 0.807). Besides, significant prognosis differences were also observed within and between such MTHFR variants after and before the specific moment during inhaled POH treatment (Supplementary Table 4) 
corroborates previous data from our group [16-18], strongly reinforcing the efficacy of $\mathrm{POH}$ inhaled therapy on prolonging rGBM patients survival for both methylation groups, achieving substantial increment for both hypo (76\%) and hypermethylated phenotypes (252\%).

The global DNA methylation status is influenced by several factors, including dietary intake of enzymatic cofactors such as B9 (folate), B12 and B6 vitamins. Such cofactors play a key role in MTHFR-mediated onecarbon metabolic pathway, thus impacting overall DNA methylation levels [46]. Genotype analysis of our rGBM patients' cohort showed that $62 \%$ of patients had at least one mutated allele for the rs1801133MTHFR gene polymorphism $(\mathrm{CT}=49 \%$; $\mathrm{TT}=13 \%)$. Important worth mentioning that reports on the role of MTHFR polymorphism in GBM were often case-control studies, mainly investigating the influence of genotypic variants on disease risk [47-49], which clearly was not the scope of the present study. Nevertheless, we are aware that caution should be taken when making comparisons between genotype frequencies among different patient populations, being imperative to consider the influence that ancestry and population heterogeneity play in each particular study.

Regardless the wide distribution of global gDNA methylation status among rGBM patients, we identified significant and negative correlations of this epigenetic signature between rGBM patients harboring the TT genotype compared to homozygous (CC genotype) variant (Figs. 2 and 3). Indeed, comparing to $\mathrm{CC}$ genotype the TT genotype showed significant reduction in gDNA methylation levels in both the hypomethylated group (2.4-fold decrease; $d=1.189$; Supplementary Table 2) and the hypermethylated group (1.2-fold decrease; $\mathrm{d}=$ 0.979, Supplementary Table 3). Interestingly, even for the hypermethylated phenotype, the homozygous TT polymorphic variant showed an expressive change in global gDNA methylation levels, being more prominent in the hypomethylated rGBM patients. Such result emphasizes the importance of the proper functionality of the key regulatory MTHFR enzyme on global DNA methylation levels.

The decrease at global DNA methylation levels observed among rGBM patients with TT genotype would suggest worse prognosis for such patients, since this mutated genotype is associated to a drastic MTHFR loss of enzymatic functionality, impacting on decreasing the global DNA methylation scores, which is described as a marker of genesis and tumor progression [3, 4, 50, 51]. Surprisingly, when considered the same survival time ( 25 weeks) from which there were observed significant differences $(d=0.639)$ between the rGBM patients survival according the phenotypes hypo (44 weeks) and hypermethylated (88 weeks; Fig. 2b, Supplementary Table 1), it was possible to identify better survival times for individuals harboring the TT rs1801133 genetic variant, despite methylation level (Supplementary Table 3). It is worth to mention the multifactorial essence of glioblastoma prognosis considering age at onset, tumor location, histology and molecular characteristics [52]. Despite of the lack of an appropriate sample size to run predictive models to study our rGBM cohort prognosis as a limitation of our study, as well as the recognized the intratumoral heterogeneity, the present results strongly demonstrates the role of gDNA global methylation and rs1801133 MTHFR variants as significant individual epigenetic and genetic candidates for rGBM patients under $\mathrm{POH}$ inhaled therapy prognosis. Besides, it seems plausible to hypothesize that due its pleiotropic nature, the monoterpene $\mathrm{POH}$ mitigates the overall impact of enzymatic MTHFR deficiency. This effect could be a result of $\mathrm{POH}$-mediated indirect effects over additional metabolic and signaling pathways [53] that combined enhanced patients' survival regardless of individual gDNA methylation status. Hence, even though decreased MTHFR activity tend to increase DNA synthesis and support rapid tumor cell proliferation, $\mathrm{POH}$ inhaled treatment could partially overcome this enzymatic deficiency promoting cell cycle arrest, apoptosis and inhibiting ROS harmful effects [19].

\section{Conclusion}

The confirmation of the hypomethylated phenotype in rGBM patients using peripheral blood cells as biological source of gDNA confirms the utility of such non-invasive approach for our patients' follow-up, since longer survival times were achieved by inhaled $\mathrm{POH}$ therapy. When compared to wild CC genotype, the homozygous TT genotype of rs1801133 (C677T) of MTHFR gene may be associated with an overall decrease in global gDNA methylation and longer survival time of rGBM patients under $\mathrm{POH}$-based therapy. Altogether, our results suggest that inhalation treatment of $\mathrm{rGBM}$ patients with $\mathrm{POH}$ mitigate the detrimental effects of global gDNA hypomethylation on tumor cell proliferation while fostering normalization of the MTHFR-mediate one-carbon folate metabolism in patients with recurrent glioblastoma harboring mutant variants for rs1801133 polymorphism. Importantly, baseline characteristics of the patient cohort showing a significant benefit from inhaled $\mathrm{POH}$ therapy ( $>25$ weeks survivors) can introduce unknown bias into the results; therefore, additional studies may be required to further validate the findings reported in this study. 


\section{Supplementary information}

Supplementary information accompanies this paper at https://doi.org/10. 1186/s12885-020-06802-8.

Additional file 1: Table S1. Comparison between Hypo and Hypermethylated patients at 25 weeks of POH treatment. Table S2. gDNA hypomethylation levels according rs1801133 (C677T) MTHFR variants. Table S3: gDNA hypermethylation levels according rs1801133 (C677T) MTHFR variants. Table S4. Prognostic relevance of rs 1801133 variants of rGBM patients at 25 weeks of $\mathrm{POH}$ treatment.

\section{Abbreviations}

GBM: Glioblastoma; gDNA: Genomic DNA; HWE: Hardy-Weinberg equilibrium; MTHFR: Methylenetetrahydrofolate reductase; $\mathrm{POH}$ : Perillyl alcohol; rGBM: Recurrent glioblastoma; rs1801131: Reference Single nucleotide polymorphism number 1801131

\section{Acknowledgements}

We thank Professor Lucio Souza Gonçalves, PhD (Faculty of Dentistry, Estacio de Sa University) for assistance with supervision with data analysis statistics.

\section{Authors' contribution}

IDPS performed experiments and draft the manuscript; GMF performed experiments, had substantial participation in the acquisition, analysis of experimental and statistical data and drafting the manuscript for important intellectual content; MDAS had substantial participation critically reviewing the manuscript for important intellectual content and for written English; BLP and COF had substantial participation on patients follow-up and manu script revision; MRA and TQ-S supervised the PhD thesis of IDPS. TQ-S conceived and designed the study, had substantial participation in the supervision, analysis, interpretation of experimental data, in the final writing and editing the manuscript, acquisition of founding and in decision to submit for publication. All authors read and approved the final version of this manuscript.

\section{Funding}

Grants for this study were provided by National Council Research (MCT/ CNPq/CT-Saude 401943/2010-0; CNPq/Universal 481059/2011-3) applicable to equipment acquisition; Euclides da Cunha Foundation-UFF supported reagents, equipment acquisition and maintenance necessary for collection, analysis and interpretation of the data. I.D.P.S had a fellowship from CAPES attached to the postgraduate program PPBI-UFF; GMF was attached to the postgraduate program of Neurologia e Neurociencias-UFF.

\section{Availability of data and materials}

The data generated and analyzed during the current study are not publicly available due confidentiality requirements, but are available from the corresponding author on reasonable request.

\section{Ethics approval and consent to participate}

All procedures were carried out with patients with recurrent GBM attending the out-patient Neurosurgical Unit in the Antonio Pedro University Hospital and enrolled on Phase I/II clinical trial to assess the efficacy of intranasal administration of the monoterpene $\mathrm{POH}$. This project was approved by Institutional and National Ethics Committees (HUAP/UFF: CAAE 0085.0.258.000-08; CONEP registration $\left.9681 \mathrm{n}^{\circ} .25000 .009267 / 2004\right)$ being also in compliance with the 1964 Helsinki Convention for clinical study and its latter amendments or comparable ethical standards. Written informed consent prior to enrollment in the clinical trial of intranasal $\mathrm{POH}$ was obtained from all individual participants included in the study.

Perillyl alcohol was formulated to be used by inhalation by the University Pharmacy in accordance with international patent application US Patent Application 20040087651 May 6, 2004 and Brazilian PI0107262-5 with approval by local Board of Health.

\section{Consent for publication}

Not applicable, since no personal information is provided in this manuscript.

\section{Competing interests}

The authors declare no conflict of interest concerning this manuscript. All authors read and approved the final version of the present manuscript

\section{Author details}

${ }^{1}$ Instituto de Biologia, Universidade Federal Fluminense, Niteroi, Rio de Janeiro, ZC 24020-141, Brazil. 'PPrograma de Pós-graduação em Neurologia, Faculdade de Medicina, Universidade Federal Fluminense, Niteroi, Rio de Janeiro 24020-141, Brazil. ${ }^{3}$ Programa de Pós-graduação em Ciencia e Biotecnologia, Universidade Federal Fluminense, Niteroi, Rio de Janeiro 24020-141, Brazil. ${ }^{4}$ Department of Hematology and HCT, City of Hope Comprehensive Cancer Center, Duarte, CA 91010, USA. ${ }^{5}$ Departamento de Medicina Especializada, Unidade de Pesquisa Clínica (UPC-HUAP), Universidade Federal Fluminense, Niteroi, RJ, Brazil.

Received: 29 January 2020 Accepted: 29 March 2020

Published online: 07 April 2020

\section{References}

1. Yang HH, Hu N, Wang C, Ding T, Dunn BK, Goldstein AM, Taylor PR, Lee MP. Influence of genetic background and tissue types on global DNA methylation patterns. PLoS One. 2010;5(2):-e9355.

2. Cadieux B, Ching T-T, VandenBerg SR, Costello JF. Genome-wide hypomethylation in human glioblastomas associated with specific copy number alteration, Methylenetetrahydrofolate Reductase allele status, and increased proliferation. Cancer Res. 2006:66(17):8469-76.

3. Cartron P-F, Hervouet E, Debien E, Olivier C, Pouliquen D, Menanteau J, Loussouarn D, A. Martin S, Campone M, Vallette FM: Folate supplementation limits the tumourigenesis in rodent models of gliomagenesis. Eur J Cancer 2012, 48(15):2431-2441.

4. Martinez R, Esteller M. The DNA methylome of glioblastoma multiforme Neurobiol Dis. 2010;39(1):40-6.

5. Körber V, Yang J, Barah P, Wu Y, Stichel D, Gu Z, Fletcher MNC, Jones D, Hentschel B, Lamszus K, et al. Evolutionary trajectories of IDHWT glioblastomas reveal a common path of early tumorigenesis instigated years ahead of initial diagnosis. Cancer Cell. 2019;35(4):692-704 e612.

6. Nishi N, Kawai S, Yonezawa T, Fujimoto K, Masui K. Early appearance of high grade glioma on magnetic resonance imaging. Neurol Med Chir. 2009:49(1):8-12.

7. Young RM, Jamshidi A, Davis G, Sherman JH. Current trends in the surgical management and treatment of adult glioblastoma. Ann Transl Med. 2015; 3(9):121.

8. Landy HJ, Lee TT, Potter P, Feun L, Markoe A. Early MRI findings in high grade glioma. J Neuro-Oncol. 2000;47(1):65-72.

9. Chang K, Zhang B, Guo X, Zong M, Rahman R, Sanchez D, Winder N, Reardon DA, Zhao B, Wen PY, et al. Multimodal imaging patterns predict survival in recurrent glioblastoma patients treated with bevacizumab. Neurooncology. 2016:18(12):1680-7.

10. Delgado-López PD, Corrales-García EM. Survival in glioblastoma: a review on the impact of treatment modalities. Clin Transl Oncol. 2016;18(11):1062-71.

11. Rajesh Y, Pal I, Banik P, Chakraborty S, Borkar SA, Dey G, Mukherjee A, Mandal M. Insights into molecular therapy of glioma: current challenges and next generation blueprint. Acta Pharmacol Sin. 2017;38(5):591-613.

12. Iwamoto F, Abrey L, Beal K, Gutin P, Rosenblum M, Reuter V, DeAngelis L, Lassman A. Patterns of relapse and prognosis after bevacizumab failure in recurrent glioblastoma. Neurology. 2009;73(15):1200-6.

13. Lemée J-M, Clavreul A, Menei P. Intratumoral heterogeneity in glioblastoma: don't forget the peritumoral brain zone. Neurooncology. 2015;17(10):1322-32.

14. Peppicelli S, Andreucci E, Ruzzolini J, Laurenzana A, Margheri F, Fibbi G, Del Rosso M, Bianchini F, Calorini L. The acidic microenvironment as a possible niche of dormant tumor cells. Cell Mol Life Sci. 2017;74(15):2761-71.

15. Qazi MA, Vora P, Venugopal C, Sidhu SS, Moffat J, Swanton C, Singh SK. Intratumoral heterogeneity: pathways to treatment resistance and relapse in human glioblastoma. Ann Oncol. 2017;28(7):1448-56.

16. Da Fonseca C, Teixeira RM, Silva JC, Fischer JS, Meirelles OC, Landeiro JA, Quirico-Santos T. Long-term outcome in patients with recurrent malignant glioma treated with Perillyl alcohol inhalation. Anticancer Res. 2013;33(12): 5625-31.

17. da Fonseca CO, Simao M, Lins IR, Caetano RO, Futuro D, Quirico-Santos T. Efficacy of monoterpene perillyl alcohol upon survival rate of patients with recurrent glioblastoma. J Cancer Res Clin Oncol. 2011;137(2):287-93. 
18. da Fonseca CO, Schwartsmann G, Fischer J, Nagel J, Futuro D, QuiricoSantos T, Gattass CR. Preliminary results from a phase I/II study of perillyl alcohol intranasal administration in adults with recurrent malignant gliomas. Surg Neurol. 2008;70(3):259-66 discussion 266-257.

19. Gomes AC, Mello AL, Ribeiro MG, Garcia DG, Da Fonseca CO, Salazar MDA, Schönthal AH, Quirico-Santos T. Perillyl alcohol, a pleiotropic natural compound suitable for brain tumor therapy, targets free radicals. Arch Immunol Ther Exp. 2017;65(4):285-97.

20. Loutrari H, Hatziapostolou M, Skouridou V, Papadimitriou E, Roussos C, Kolisis FN, Papapetropoulos A. Perillyl alcohol is an angiogenesis inhibitor. J Pharmacol Exp Ther. 2004;311(2):568-75.

21. Pavlova NN, Thompson CB. The emerging hallamarks of cancer metabolism. Cell Metab. 2016;23(1):27-47.

22. Carlsson SK, Brothers SP, Wahlestedt C. Emerging treatment strategies for glioblastoma multiforme. EMBO Mol Med. 2014;6(11):1359-70.

23. Cheng T-YD, Makar KW, Neuhouser ML, Miller JW, Song X, Brown EC, Beresford SAA, Zheng Y, Poole EM, Galbraith RL, et al. Folate-mediated onecarbon metabolism genes and interactions with nutritional factors on colorectal cancer risk: Women's Health Initiative observational study. Cancer. 2015;121(20):3684-91.

24. Kinnaird A, Zhao S, Wellen KE, Michelakis ED. Metabolic control of epigenetics in cancer. Nat Rev Cancer. 2016;16:694.

25. Newman AC, Maddocks ODK. One-carbon metabolism in cancer. $\mathrm{Br} \mathrm{J}$ Cancer. 2017;116(12):1499-504.

26. Alexander BM, Cloughesy TF. Adult Glioblastoma. J Clin Oncol. 2017;35(21): 2402-9.

27. Frosst $P$, Blom HJ, Milos R, Goyette $P$, Sheppard CA, Matthews RG, Boers GJH, den Heijer M, Kluijtmans LAJ, van den Heuve LP, et al. A candidate genetic risk factor for vascular disease: a common mutation in methylenetetrahydrofolate reductase. Nat Genet. 1995;10(1):111-3.

28. Raichle ME. The restless brain: how intrinsic activity organizes brain function. Philos Trans R Soc Lond B Biol Sci. 2015;370(1668):20140172.

29. Faria G, Silva E, Da Fonseca C, Quirico-Santos T. Circulating cell-free DNA as a prognostic and molecular marker for patients with brain tumors under perillyl alcohol-based therapy. Int J Mol Sci. 2018;19(6):1610.

30. Wang J, Bettegowda C. Applications of DNA-based liquid biopsy for central nervous system neoplasms. J Mol Diagn. 2017;19(1):24-34.

31. Zukiel R, Nowak S, Barciszewska A-M, Gawronska I, Keith G, Barciszewska MZ. A simple epigenetic method for the diagnosis and classification of brain Tumors11Polish Committee of Scientific Research Project (M. Barciszewska). Mol Cancer Res. 2004;2(3):196-202.

32. Jones MJ, Goodman SJ, Kobor MS. DNA methylation and healthy human aging. Aging Cell. 2015;14(6):924-32.

33. Parker NR, Hudson AL, Khong P, Parkinson JF, Dwight T, Ikin RJ, Zhu Y, Cheng ZJ, Vafaee F, Chen J. Intratumoral heterogeneity identified at the epigenetic, genetic and transcriptional level in glioblastoma. Sci Rep. 2016;6:22477.

34. Mutchinick OM, MaA L, Luna L, Waxman J, Babinsky VE, Group RC. High prevalence of the thermolabile methylenetetrahydrofolate reductase variant in Mexico: a country with a very high prevalence of neural tube defects. Mol Genet Metab. 1999;68(4):461-7.

35. Sullivan GM, Feinn R. Using effect size-or why the $P$ value is not enough. J Graduate Med Educ. 2012:4(3):279-82.

36. Lenhard WL, A. Calculation of effect sizes. Dettelbach: Psychometrica. 2016. Retrieved from: https://www.psychometrica.de/effect_size.html. https://doi. org/10.13140/RG.2.2.17823.92329.

37. Schober P, Boer C, Schwarte LA. Correlation coefficients: appropriate use and interpretation. Anesth Analg. 2018;126(5):1763-8.

38. Barciszewska A-M, Nowak S, Naskręt-Barciszewska MZ. The degree of global DNA hypomethylation in peripheral blood correlates with that in matched tumor tissues in several neoplasia. PLoS One. 2014;9(3):-e92599.

39. Kuchiba A, Iwasaki M, Ono H, Kasuga Y, Yokoyama S, Onuma H, Nishimura H, Kusama R, Tsugane S, Yoshida T. Global methylation levels in peripheral blood leukocyte DNA by LUMA and breast cancer: a case-control study in Japanese women. Br J Cancer. 2014;110(11):2765-71.

40. Miranda-Morales E, Meier K, Sandoval-Carrillo A, Salas-Pacheco J, VázquezCárdenas P, Arias-Carrión O. Implications of DNA methylation in Parkinson's disease. Front Mol Neurosci. 2017;10:225.

41. Klekner Á, Szivos L, Virga J, Árkosy P, Bognár L, Birkó Z, Nagy B. Significance of liquid biopsy in glioblastoma-A review. J Biotechnol. 2019;298:82-7.

42. Hanahan D, Weinberg RA. Hallmarks of cancer: the next generation. Cell. 2011;144(5):646-74.
43. Netea-Maier RT, Smit JW, Netea MG. Metabolic changes in tumor cells and tumor-associated macrophages: A mutual relationship. Cancer Lett. 2018; 413:102-9.

44. Wishart DS. Metabolomics for investigating physiological and pathophysiological processes. Physiol Rev. 2019;99(4):1819-75.

45. Kondo Y, Katsushima K, Ohka F, Natsume A, Shinjo K. Epigenetic dysregulation in glioma. Cancer Sci. 2014;105(4):363-9.

46. Krushkal J, Zhao Y, Hose C, Monks A, Doroshow JH, Simon R. Concerted changes in transcriptional regulation of genes involved in DNA methylation, demethylation, and folate-mediated one-carbon metabolism pathways in the $\mathrm{NCl}-60$ cancer cell line panel in response to cancer drug treatment. Clin Epigenetics. 2016:8:73.

47. Bethke L, Webb E, Murray A, Schoemaker M, Feychting M, Lonn S, Ahlbom A, Malmer B, Henriksson R, Auvinen A, et al. Functional polymorphisms in folate metabolism genes influence the risk of meningioma and glioma. Cancer Epidemiol Biomark Prev. 2008;17(5):1195-202.

48. Da Costa DM, De Lima GPV, Faria MHG, Rabenhorst SHB. Polymorphisms of folate pathway enzymes (Methylenetetrahydrofolate Reductase and Thymidylate synthase) and their relationship with thymidylate synthase expression in human astrocytic tumors. DNA Cell Biol. 2012;31(1):57-66.

49. Izmirli M. A literature review of MTHFR (C677T and A1298C polymorphisms) and cancer risk. Mol Biol Rep. 2013:40(1):625-37.

50. Stern LL, Mason JB, Selhub J, Choi S-W. Genomic DNA hypomethylation, a characteristic of most cancers, is present in peripheral leukocytes of individuals who are homozygous for the C677T polymorphism in the Methylenetetrahydrofolate Reductase gene. Cancer Epidemiol Biomark Prev. 2000;9(8):849-53.

51. Weisberg I, Tran P, Christensen B, Sibani S, Rozen R. A second genetic polymorphism in Methylenetetrahydrofolate Reductase (MTHFR) associated with decreased enzyme activity. Mol Genet Metab. 1998;64(3):169-72.

52. Louis DN, Perry A, Reifenberger G, von Deimling A, Figarella-Branger D, Cavenee WK, Ohgaki H, Wiestler OD, Kleihues P, Ellison DW. The 2016 World Health Organization classification of tumors of the central nervous system: a summary. Acta Neuropathol. 2016;131(6):803-20.

53. Gomes AC, Mello AL, Ribeiro MG, Garcia DG, Da Fonseca CO, Salazar MD, Schonthal AH, Quirico-Santos T. Perillyl alcohol, a pleiotropic natural compound suitable for brain tumor therapy, targets free radicals. Arch Immunol Ther Exp. 2017;65(4):285-97.

\section{Publisher's Note}

Springer Nature remains neutral with regard to jurisdictional claims in published maps and institutional affiliations.
Ready to submit your research? Choose BMC and benefit from:

- fast, convenient online submission

- thorough peer review by experienced researchers in your field

- rapid publication on acceptance

- support for research data, including large and complex data types

- gold Open Access which fosters wider collaboration and increased citations

- maximum visibility for your research: over $100 \mathrm{M}$ website views per year

At BMC, research is always in progress.

Learn more biomedcentral.com/submissions 